\title{
Integrals of functions containing parameters
}

Robert M. Corless, David J. Jeffrey and David R. Stoutemyer

\section{Introduction}

"There is always a well-known solution to every human problem - neat, plausible, and wrong."

H. L. Mencken 15$]^{1}$

The Non Sequitur cartoon for 20 January 2016

Calculus students are taught that an indefinite integral is defined only up to an additive constant, and as a consequence generations of students have assiduously added " $+C$ " to their calculus homework. Although ubiquitous, these constants rarely garner much attention, and typically loiter without intent around the ends of equations, feeling neglected. There is, however, useful work they can do; work which is particularly relevant in the contexts of integral tables and computer algebra systems. We begin, therefore, with a discussion of the context, before returning to coax the constants out of the shadows and assign them their tasks.

Tables of integrals are inescapable components of calculus textbooks [2, 18, and there are well known reference books that publish voluminous collections [1, 9, 16, 20. A modern alternative to integral tables is provided by computer algebra systems (CAS), which are readily available on computing platforms ranging from phones to supercomputers. These systems evaluate integrals using a mixture of integral tables and algebraic algorithms. A feature shared by tables and computer systems is the fact that the formulae usually contain parameters. No one would want a table of integrals that contained separate entries for $x, x^{2}$ and $x^{42}$, rather than one entry for $x^{n}$, and many tables include additional parameters for user convenience; for example, there will be entries for integrals containing sin $a x$, rather than the sufficient, but less convenient, $\sin x$.

Although parameters add greatly to the scope and convenience of integral tables, there can be difficulties and drawbacks occasioned by their use. We shall use the word specialization to describe the action of substituting specific values (usually numerical, but not necessarily) into a formula. The specialization problem is a label for a cluster of

\footnotetext{
${ }^{1}$ The truth of this statement is reinforced by the fact that it is often misquoted.
} 
issues associated with formulae and their specialization, the difficulties ranging from inelegant results to invalid ones. For example, in [12] an example is given in which the evaluation of an integral by specializing a general formula misses a particular case for which a more elegant expression is possible. The focus here, however, is on situations in which specialization leads to invalid or incorrect results. To illustrate the problems, consider an example drawn from a typical collection [19, ch8, p346, (5)]:

$$
I_{1}=\int\left(\alpha^{\sigma z}-\alpha^{\lambda z}\right)^{2} d z=\frac{1}{2 \ln \alpha}\left(\frac{\alpha^{2 \lambda z}}{\lambda}+\frac{\alpha^{2 \sigma z}}{\sigma}-\frac{4 \alpha^{(\lambda+\sigma) z}}{\lambda+\sigma}\right)
$$

Expressions equivalent to this are returned by Maple, Mathematica and many other systems, such as the Matlab symbolic toolbox.

Before we proceed, we acknowledge that some readers may question whether anyone at all competent would write the integral this way: surely there are better ways? Why not transform $\alpha^{\sigma z}$ into $\exp (p z)$, where $p=\sigma \ln \alpha$, and thus reduce the number of parameters? Or scale the variable of integration to absorb, say, the $\lambda$ ? Such actions are possible for people who are free to recast problems in convenient ways, for example, if (1.1) were an examination question devoid of context. CAS, in contrast, are obliged to deal with expressions as they are presented, either by users or by other components within the system itself; and in the general case some of these "obvious" simplifications and transformations are surprisingly difficult to discover automatically. Humans are still superior at simplification, we believe.

Returning to the answer as returned by the CAS, it is easy to see that the specialization $\sigma=0$ leaves the left side of (1.1), the integrand, well defined, but the expression for its integral on the right-hand side is no longer defined. If we pursue this further, we see that there are multiple specializations for which (1.1) fails, viz. $\alpha=0, \alpha=1, \lambda=0$, $\sigma=0, \lambda=-\sigma$, and combinations of these. The question of how or whether to inform computer users of these special cases has been discussed in the CAS literature many times [6].

This brings us to the second theme of this discussion: comprehensive and generic results. A comprehensive result lists explicit expressions for each set of special parameter values, while a generic result is correct for 'most' or 'almost all' values of the parameters. Let us consider how a comprehensive result for (1.1) would look. 


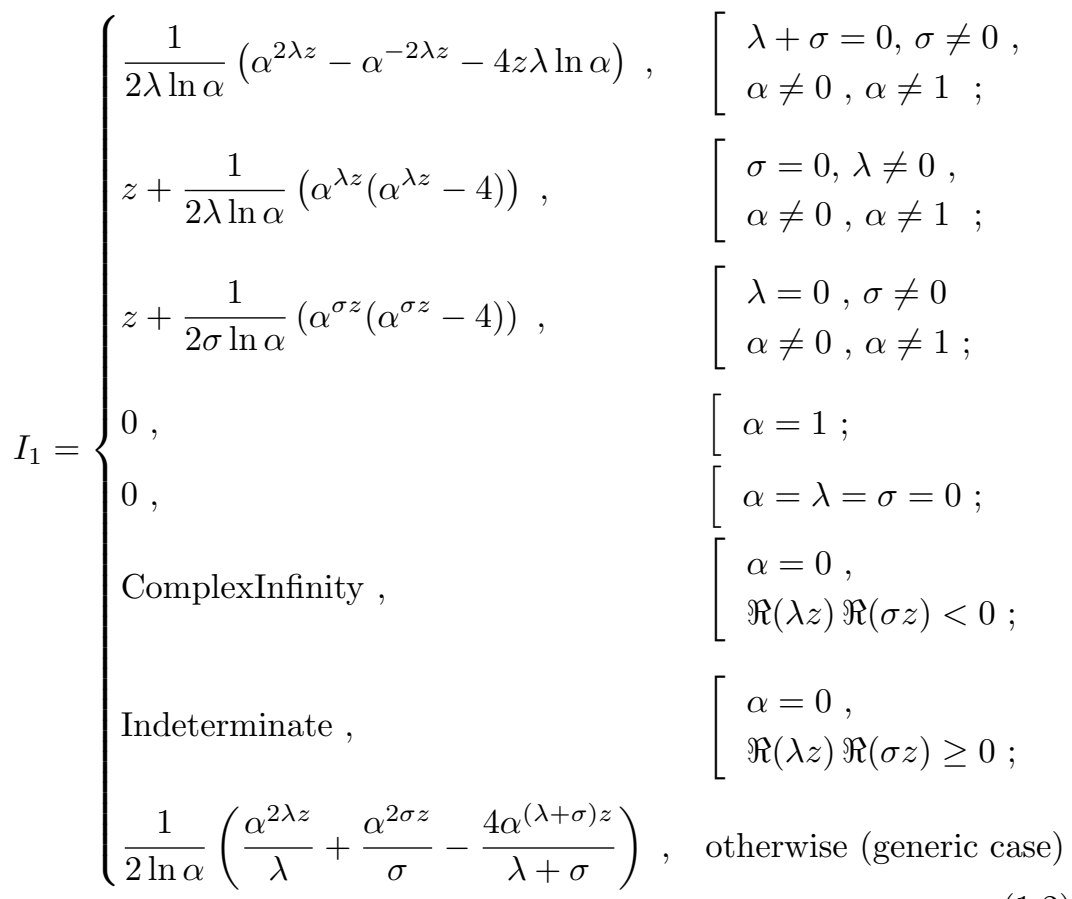

Conditions are here shown as in printed tables; otherwise they could be presented using the logical $\vee$ and $\wedge$ operators.

To generalize, we denote a function depending on parameters by $f(z ; \mathbf{p})$, with $z$ being the main argument, here the integration variable, and $\mathbf{p}$ a list of parameters. The definition is then:

Definition 1.1 A comprehensive antiderivative of a parametric function $f(z ; \mathbf{p})$ is a piecewise function $F(z ; \mathbf{p})$ containing explicit consequent ${ }^{2}$ for each special case of the parameters.

Designers of computer algebra systems are reluctant to return comprehensive expressions by default, because they can quickly lead to unmanageable computations, and as well many users might regard them as too much information. Instead, tables and CAS commonly adopt the approach of identifying a generic case, which is then the only expression given; in the case of CAS, the generic case is typically returned without explicitly showing the conditions on the parameters. In the

\footnotetext{
${ }^{2}$ consequent: following as a result or effect; the second part of a conditional proposition, dependent on the antecedent.
} 
case of tables, any special-case values would be used to simplify the integrand and then the resulting integrand and its antiderivative would be displayed as a separate entry somewhere else in the table.

Definition 1.2 A generic antiderivative is one expression chosen from a comprehensive antiderivative that is valid for the widest class of constraints.

Remark 1.3 The above definition is an informal one, since the choice of which result to designate as generic may include personal taste.

\section{Continuity in parameters}

We now come to the third theme of the discussion: the treatment of removable discontinuities. Consider the improper integral

$$
\int_{0}^{1} \ln (x(1-x)) d x=[x \ln (x(1-x))-2 x-\ln (1-x)]_{0}^{1} .
$$

The expression for the integral contains a removable discontinuity at each end, and a computer system (and we hope students) would automatically switch to limit calculations to obtain the answer. In this section, by extending the handling of removable discontinuities to constants of integration, we introduce a new idea for handling the specialization problem in integration ${ }^{3}$. The idea is new in the sense that we do not know of any published discussion, but it originated with William Kahan [14] and was circulated informally possibly as early as 1959.

The example 1.2 dramatically illustrates the potential size of comprehensive antiderivatives, but is, unsurprisingly, too cumbersome for explaining ideas. We turn to simpler examples. We begin with the comprehensive antiderivative known to all students of calculus 4 .

$$
\int z^{\alpha} \mathrm{d} z= \begin{cases}\ln z, & \text { if } \alpha=-1 \\ \frac{z^{\alpha+1}}{\alpha+1}, & \text { otherwise (generic case) }\end{cases}
$$

Substituting $\alpha=-1$ into the generic case gives $1 / 0$ and not $\ln z$. Often when a substitution fails, a limit will succeed, so we try the limit as

\footnotetext{
${ }^{3}$ The specialization problem is not confined to integration. Any formula which uses parameters to cover multiple cases is likely to have some specialization problems. The ideas presented here, however, apply specifically to integration.

${ }^{4}$ Most textbooks use $x^{n}$, but we wish to emphasize continuity and use $\alpha$ instead of $n$. We also use $z$ and $\alpha$ because we are thinking in the complex plane, and we prefer $\ln z$ instead of $\ln |x|$ for the same reason.
} 
$\alpha \rightarrow-1$. Disappointingly, this also fails, but we can examine how the limit fails by expanding the generic case as a series about the pole at $\alpha=-1$, that is, treating $\alpha+1=\varepsilon$ as a small quantity.

$$
\frac{z^{\alpha+1}}{\alpha+1}=\frac{e^{\varepsilon \ln z}}{\varepsilon}=\frac{1+\varepsilon \ln z+O\left(\varepsilon^{2}\right)}{\varepsilon} .
$$

If we can remove the leading term of the series, namely $1 / \varepsilon$, then the next term gives us the desired $\ln z$. But an integral needs a constant $t^{5}$ So, an equally correct integral is

$$
\int z^{\alpha} \mathrm{d} z=\frac{z^{\alpha+1}}{\alpha+1}-\frac{1}{\alpha+1}
$$

and now the limit as $\alpha \rightarrow-1$ is precisely $\ln z$. Thus the comprehensive antiderivative,

$$
\int z^{\alpha} \mathrm{d} z= \begin{cases}\ln z, & \text { if } \alpha=-1 \\ \frac{z^{\alpha+1}-1}{\alpha+1}, & \text { otherwise. }\end{cases}
$$

is continuous with respect to $\alpha$, and the generic antiderivative now contains the exceptional case as a removable discontinuity [14.

Definition 2.1 Let a function $F(z ; \mathbf{p})$ be an indefinite integral of an integrand $f(z ; \mathbf{p})$. That is,

$$
F(z ; \mathbf{p})=\int f(z ; \mathbf{p}) \mathrm{d} z .
$$

If a point $\mathbf{p}_{c}$ in parameter space exists at which $F(z ; \mathbf{p})$ is discontinuous with respect to one or more members of $\mathbf{p}$, and if a function $C(\mathbf{p})$, which serves as a constant of integration with respect to $z$, has the property that $F(z ; \mathbf{p})+C(\mathbf{p})$ has only a removable discontinuity and thus can be made continuous with respect to $\mathbf{p}$ at $\mathbf{p}_{c}$, then $C(\mathbf{p})$ is called a Kahanian constant of integration ${ }^{6}$

Remark 2.2 The definition does not guarantee the existence of $C(\mathbf{p})$. If, for some value of $\mathbf{p}$, the integral does not exist, then there will be no Kahanian.

\footnotetext{
${ }^{5}$ The lack of a constant in $\sqrt{2.2}$ betrays our CAS allegiance: it is a rare CAS that adds a constant, because the user can easily add one (and name the constant) by typing, for instance, "int $(f, x)+K$ ".

${ }^{6}$ Since it is a function of $\mathbf{p}$, one can question whether it should be called a constant. It is constant with respect to $z$, and this seems a useful extension of calculus terminology.
} 
Figure 1: A parametrically discontinuous integral. The real part of each consequent of the comprehensive integral $(2.5)$ is plotted. The surface shows the generic expression plotted as a function of the integration variable $z$ and the parameter $\alpha$. The surface curves up to become singular all along the line $\alpha=0$. The detached curve hovering below the surface in the plane $\alpha=0$ is the special case integral.

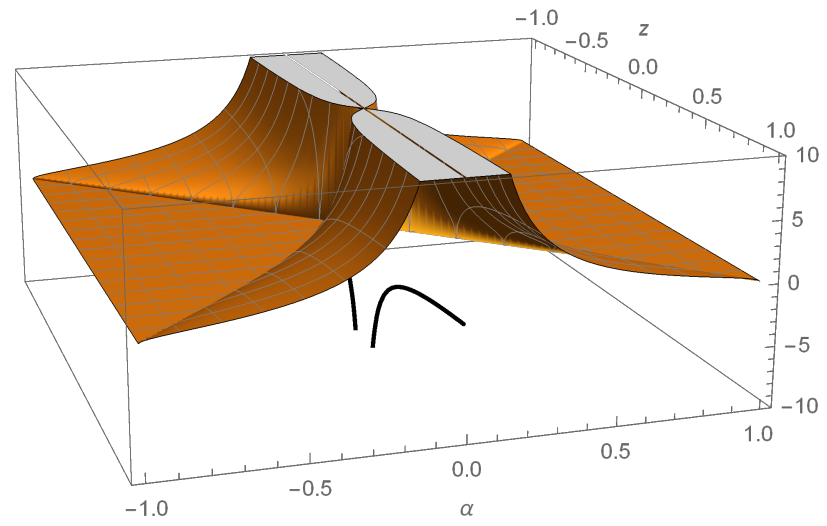

A second example shows the effect of Kahanian constants graphically. To begin with, consider the comprehensive antiderivative

$$
\int \frac{d z}{z \sqrt{z^{2}-\alpha^{2}}}= \begin{cases}-\frac{1}{\sqrt{z^{2}}}, & \alpha=0, \\ \frac{1}{\alpha} \operatorname{arccot} \frac{\alpha}{\sqrt{z^{2}-\alpha^{2}}}, & \text { generic . }\end{cases}
$$

We write $\sqrt{z^{2}}$ rather than $|z|$ so that the expression is valid for nonreal $z$. Figure 1 shows this antiderivative as a three-dimensional plot treating both $z$ and $\alpha$ as real variables. The generic expression is then the surface shown in the plot; it becomes singular along the line $\alpha=0$ (as $\alpha^{-2}$ ). The special case $\alpha=0$ is shown as a detached curve confined to the plane $\alpha=0$. It can be seen hovering forlornly underneath the surface of the generic integral, dreaming of gaining an invitation to the party. For $|z|<|\alpha|$, the values of the integral are non-real, but only the real part is plotted, because that displays the properties of interest 7 .

\footnotetext{
${ }^{7}$ The definition of arccot varies between computer systems and amongst references, and even between different printings of the same reference work [7]. The plots shown here were made with Mathematica, and other systems such as Maple and Matlab may create different plots.
} 
Figure 2: A parametrically continuous integral. The real part of each consequent of the comprehensive integral (2.6) is plotted. The surface shows the generic expression plotted as a function of the integration variable $z$ and the parameter $\alpha$. The surface curves down, and is singular only at $z=\alpha=0$. The special case curve lies happily within the surface in the plane $\alpha=0$.

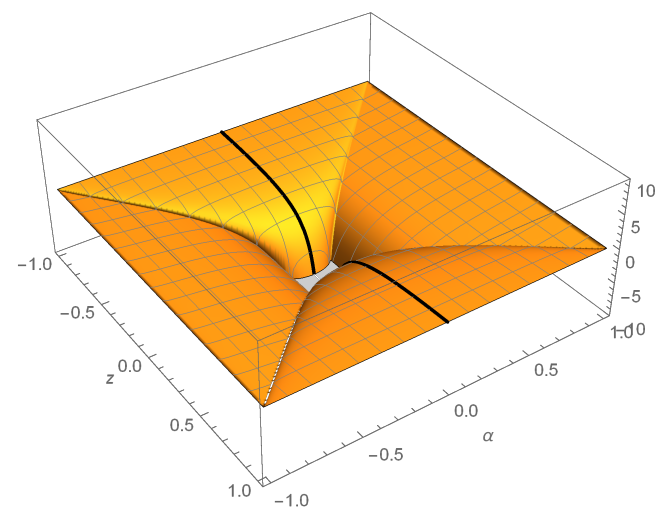

To achieve continuity, we now add Kahanian constants to each consequent of the comprehensive antiderivative. We show the new constants in bold print.

$$
\int \frac{d z}{z \sqrt{z^{2}-\alpha^{2}}}=\left\{\begin{array}{l}
-\frac{1}{\sqrt{z^{2}}}+\mathbf{1} \\
\frac{1}{\alpha} \operatorname{arccot} \frac{\alpha}{\sqrt{z^{2}-\alpha^{2}}}-\frac{1}{\alpha} \operatorname{arccot} \frac{\alpha}{\sqrt{1-\alpha^{2}}}
\end{array}\right.
$$

Figure 2 shows the real part of the new expressions. The extra term in the generic (the lower) consequent in (2.6) subtractively cancels a parametric pole asymptotic to $1 / \alpha^{2}$ as $\alpha \rightarrow 0$, which can be seen in the generic consequent of result 2.5. This makes the parametrically continuous expression now approach the same values from both sides of $\alpha=0$, converting that parametric pole to an indeterminate slit in an otherwise parametrically continuous surface - a removable singularity. Moreover, the extra term 1 in the $\alpha=0$ consequent of (2.6) raises the space curve exactly the right amount to make it contiguous with the surface on both sides, thus removing that removable singularity. 


\section{Advantages of parametrically continuous integrals}

We have defined the specialization problem as the failure of a generic formula when particular values are substituted for parameters, with the generic integrals above being examples. One way to avoid specialization problems completely would be to specify all parameters in advance, that is, delay starting a calculation until the parameters are known. This, however, negates the very power and generality that algebra extends to us. As well, it is not always the case that one can know parameters in advance; for example, a parameter might depend on the outcome of an intermediate computation.

The specialization problem can be looked at another way, a way perhaps more suitable for computer algebra. There are variations possible in the order in which operations are applied. For example, in Maple syntax, where as usual operations take precedence from the inside out, it is the difference between

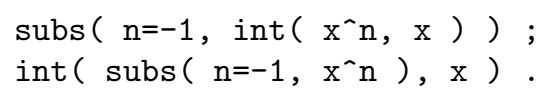

The first gives a division by zero error; the second gives $\ln x$. The challenge, then, is to retain the generality of algebra, while finding ways to react to exceptional values of parameters.

\subsection{Definite integration}

There are at least two ways to evaluate a special case of a known parametric definite integral, especially in the computer-algebra setting. The first way is the typical human way: by working on each limit separately. Thus to evaluate

$$
\int_{a}^{b} f(x, \mathbf{p}) \mathrm{d} x
$$

one first finds an indefinite integral $F(x, \mathbf{p})$, then makes any substitutions $F\left(x, \mathbf{p}_{c}\right)$, then evaluates and simplifies $F\left(b, \mathbf{p}_{c}\right)-F\left(a, \mathbf{p}_{c}\right)$. For this approach, the Kahanian term is important, because it allows a sub-

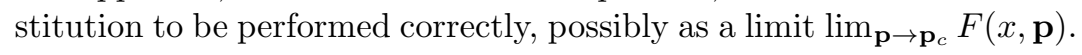

For the second way, let us explicitly notate the presence of a Kahanian term, so that the indefinite integral is $F(x, \mathbf{p})+C(\mathbf{p})$, where now $F$ is any function that satisfies $F^{\prime}=f$ and $C(\mathbf{p})$ is the Kahanian 
constant. We now perform the definite integral before specializing.

$$
\begin{aligned}
\int_{a}^{b} f(x, \mathbf{p}) \mathrm{d} x & =[F(b, \mathbf{p})+C(\mathbf{p})]-[F(a, \mathbf{p})+C(\mathbf{p})] \\
& =F(b, \mathbf{p})-F(a, \mathbf{p}) .
\end{aligned}
$$

Now we must evaluate this expression as $\mathbf{p} \rightarrow \mathbf{p}_{c}$. If we simplify each term separately, the calculation may fail, but keeping the terms together, the calculation succeeds.

For example,

$$
\lim _{n \rightarrow-1} \int_{a}^{b} x^{n} \mathrm{~d} x=\lim _{n \rightarrow-1}\left(\frac{b^{n+1}}{n+1}-\frac{a^{n+1}}{n+1}\right)=\ln b-\ln a,
$$

using the methods of 2.3. Again we point out that separately the limits of each term need not exist. Computer systems, like people, can sometimes succeed when asked one way and not another. With the Kahanian form, either approach succeeds.

\subsection{Resonance}

We now give an example of Kahanian terms used in the solution of a differential equation. A standard topic in physics and engineering is resonance. The equation of a forced, frictionless, harmonic oscillator is

$$
\frac{d^{2} x(t)}{d t^{2}}+k^{2} x(t)=\cos \omega t
$$

It has the generic general solution

$$
x(t)=C_{1} \cos k t+C_{2} \sin k t+\frac{\cos \omega t}{k^{2}-\omega^{2}},
$$

where $C_{1}$ and $C_{2}$ depend on initial conditions, and the last term is the particular integral. The phenomenon of resonance occurs when $\omega^{2}=k^{2}$ and the particular integral becomes invalid. We shall now derive a particular integral containing Kahanian terms that enable the particular integral to have a valid limit in the resonant case. We shall use the method of variation of parameters [4, 13. We start from the solutions to the homogeneous equation: $x_{1}=\cos k t$ and $x_{2}=\sin k t$. Then the particular integral is given by $x_{p}=u_{1} x_{1}+u_{2} x_{2}$, where

$$
u_{1}=-\int \frac{x_{2} \cos \omega t}{W} \mathrm{~d} t, \quad u_{2}=\int \frac{x_{1} \cos \omega t}{W} \mathrm{~d} t,
$$


and $W=x_{1} x_{2}^{\prime}-x_{2} x_{1}^{\prime}$ is the Wronskian. Evaluating the integrals in the usual way, we obtain

$$
\begin{aligned}
& u_{1}=\frac{\cos ((k-w) t)}{2 k(k-w)}+\frac{\cos ((k+w) t)}{2 k(k+w)}, \\
& u_{2}=\frac{\sin ((k-w) t)}{2 k(k-w)}+\frac{\sin ((k+w) t)}{2 k(k+w)} .
\end{aligned}
$$

When the expression for $x_{p}$ is simplified, we are led to (3.3). If, however, we change to Kahanian antiderivatives, we obtain

$$
u_{1}=\frac{\cos ((k-w) t)-1}{2 k(k-w)}+\frac{\cos ((k+w) t)-1}{2 k(k+w)},
$$

and no Kahanian is needed for $u_{2}$, so it is still given by (3.5). The new particular integral is

$$
x_{p}(t)=\frac{\cos \omega t-\cos k t}{k^{2}-\omega^{2}},
$$

and now the limits $\omega \rightarrow \pm k$ give

$$
x_{p}(t)=\frac{t}{2 k} \sin k t,
$$

showing an oscillation that increases with time - a hallmark of resonant behaviour 8

Both Mathematica and Maple return (3.3) without provisos, and to obtain (3.8), one must substitute $\omega=k$ and rerun the solution. The lesson from this example is that, because of their black-box automation, computer algebra systems should implement and exploit comprehensive results from the most basic operations on up through the most sophisticated. For example, it would greatly help to have comprehensive limits and comprehensive series, as well.

\section{Computing a Kahanian antiderivative}

The derivation used in (2.4) appears ad hoc, but a more systematic procedure is possible. Instead of computing an indefinite integral, we calculate a "semi-definite" integral.

\footnotetext{
${ }^{8}$ This is an example of a computation that ought to be routine, for a human using familiar trigonometric identities. Instead of thinking back to how you solved it in your first course in differential equations, correctly accounting for resonance, imagine that you are a computer subroutine, having to turn out a good answer by a mechanical algorithm. In that context, the Kahanian approach makes automation easier.
} 
Definition 4.1 A parametric semidefinite integral is one of the form

$$
P(z ; \mathbf{p})=\int_{A}^{z} f(\tau ; \mathbf{p}) \mathrm{d} \tau,
$$

where the lower limit $A$ is called the anchor point, and is constant.

Remark 4.2 Some people use the term indefinite integral to describe (4.1), allowing one point (usually the lower limit) to be fixed and the other to vary. We, however, have earlier used the term indefinite integral to mean any antiderivative or primitive, as is common parlance where we work. To avoid confusion and to fix attention on the anchor point, we have introduced the term "semi-definite", although we are somewhat indefinite (that is, semi-indefinite) about the hyphen in the term.

Lemma 4.3 In 4.1), let $z$ be finite and let $f(\tau ; \mathbf{p})$ be continuous with respect to $\mathbf{p}$ and with respect to $\tau$ in the domain of interest containing $z, \tau$ and $A ; A$ is a fixed finite numeric constant. Then $P(z ; \mathbf{p})$ is continuous with respect to all finite values of its parameters $\mathbf{p}$, except perhaps for removable singularities.

Proof 4.4 This theorem is a consequence of classical theorems about the interchange of limits when functions are uniformly continuous: see for instance [3]. As a conceptual alternative, consider the following. Let $G(z ; \mathbf{p})$ be a generic antiderivative of $f(z ; \mathbf{p})$. Then

$$
P(z ; \mathbf{p})=G(z ; \mathbf{p})-G(A ; \mathbf{p}),
$$

and all discontinuities whose locations depend only on the parameters occur both in $G(z ; \mathbf{p})$ and in $G(A ; \mathbf{p})$. Therefore they cancel in the semidefinite integral (4.2), leaving at worst an expression that is indeterminate at the locations of those discontinuities, making the discontinuities removable.

Remark 4.5 The finiteness of the interval in the hypotheses is necessary. If the interval of integration is unbounded, then the lemma need not be true. For example, for real $x$ we have (the signum function is -1 for negative arguments, +1 for positive arguments, and zero for zero argument)

$$
\int_{-\infty}^{x} \frac{\sin p t}{t} d t=\frac{\pi}{2} \operatorname{signum}(p)+\int_{0}^{p x} \frac{\sin u}{u} d u
$$

which has a jump discontinuity at $p=0$ although the integrand $\sin (p t) / t$ is continuous there. We are indebted to a referee for this example. 
Remark 4.6 The complexity of the Kahanian depends on the choice of anchor point $A$. We want to avoid values of $A$ that make $G(A ; \mathbf{p})$ indeterminate or take an infinite magnitude. The least complex Kahanian is 0 . Therefore it is worth comparing the complexities of Kahanian constants corresponding to different anchor points, and preferring any that yield a Kahanian of 0 .

\subsection{Earlier examples}

The example 2.4 is obtained from the semidefinite integral $\int_{1}^{z} \tau^{\alpha} \mathrm{d} \tau$. Our opening example (1.1) can be modified by using an anchor point $A=0$ to calculate a Kahanian constant: we obtain

$$
\begin{aligned}
& \int\left(\alpha^{\sigma z}-\alpha^{\lambda z}\right)^{2} d z= \\
& \quad \frac{-(\boldsymbol{\lambda}-\sigma)^{2}}{2 \boldsymbol{\lambda} \boldsymbol{\sigma}(\boldsymbol{\lambda}+\boldsymbol{\sigma}) \ln \boldsymbol{\alpha}}+\frac{1}{2 \ln \alpha}\left(\frac{\alpha^{2 \lambda z}}{\lambda}+\frac{\alpha^{2 \sigma z}}{\sigma}-\frac{4 \alpha^{(\lambda+\sigma) z}}{\lambda+\sigma}\right) .
\end{aligned}
$$

It is straightforward to verify that by taking the limits $\alpha \rightarrow 0, \lambda \rightarrow 0$ and $\lambda \rightarrow-\sigma$, each of the special cases in 1.2 is reproduced. That is, the use of a Kahanian makes each of the special cases of the comprehensive integral into a removable discontinuity. Therefore, the conceptual advance of replacing evaluation using substitution by evaluation using limits, as discussed previously, can be usefully applied.

\section{Implications}

The danger of exceptional values is as old as algebra. Early enthusiasts, amazed at algebraic power, often overlooked exceptions. Eventually, though, experts such as Cauchy and Weierstrass worked to check the enthusiasm, pointing out that care was needed. Out of this care, modern analysis was born; out of the enthusiasm, modern algebra. Hawkins [10] writes of Cauchy

"Nevertheless, Cauchy did not accept the particular algebraic foundation used by Lagrange ... Cauchy, however, had well-founded doubts about the automatic general interpretation of symbolic expressions. He had warned that "most (algebraic) formulas hold true only under certain conditions, and for certain values of the quantities they contain." 
The dichotomy between analysis and algebra survives to this day, and can be seen in the present discussion of integration. Within computer algebra, integration is based on modern algebraic algorithms, such as the Risch algorithm and its generalizations [17, 5, for solving the elementary antidifferentiation problem. Algebraic algorithms explicitly exempt the constant of integration from consideration. Indeed, when verifying an integration formula by differentiation, all piecewise constants are given zero derivatives.

\subsection{Computer algebra system design}

In the early days of computer algebra, implementers and users were equally delighted at the ability of systems to obtain generic results. Now, however, the low-hanging fruit has been harvested, and implementers can and should make another pass through their fundamental functionalities such as integration, the solving of systems of equations and inequalities, limits and series to make as many results as possible comprehensive. The robustness of high-level functionality demands it.

\subsection{Mathematical tables}

Computer algebra and the internet make it decreasingly likely that there will be a completely new printed table of integrals. There will, however, probably be new editions of existing tables, because there is something to be learned by scanning a table of closely-related integrals, as opposed to seeing results one at a time from a computer algebra system, with no organizing principle.

Printed integral tables would be impractically bulky if every entry were a piecewise result of the kind in this article. However, many of the special cases could be listed once in the most appropriate place, then explicitly cross referenced from more generic cases. New editions can also make the relevant domains as general as possible and more explicitly obvious.

Moreover, an on-line version could assemble each piecewise result as needed. There could even be a computer algebra system involved. The difference of such a mathematical knowledge base from a bare computer algebra system is that a user can learn by browsing through related examples that follow a clear organizing principle. 


\subsection{Mathematical practice}

"A lot of times, people don't know what they want until you show it to them."

-Steve Jobs

Mathematicians are increasingly frequent users of computer algebra and other educational or research mathematical software. We suspect that when users start encountering more comprehensive results, they will become disappointed in software that does not provide them. As a side benefit, users might become more careful about not overlooking special cases or relevant issues such as domain enforcement and continuity issues with their manually derived results. Perhaps editors and referees will also pay more attention to such details in articles they are reviewing.

Meanwhile we hope that this article serves as a warning that when a computer algebra system returns a generic parametric antiderivative, the user should ponder the result, to determine whether there exist special cases, and if so, compute them with separate integrands.

We think that the idea of comprehensive antiderivatives will be welcomed by many mathematicians, perhaps after they are exposed to it through using future versions of computer algebra systems that offer built-in comprehensive antiderivatives. We admit, however, that parametrically continuous antiderivatives will be adopted only slowly, because they are not always necessary, and they are usually more complicated than the simpler, incorrect answer. In addition, from a numerical point of view, they can suffer from catastrophic numerical cancellation, which requires higher precision (or perhaps some numerical analysis experience) to overcome. Nonetheless, the point remains that when they are necessary, they are crucial to obtaining a correct and complete result.

\subsection{Implications for mathematics education}

The calculus curriculum is already quite full, and it seems unlikely that the textbook examples and the expected exercise or test results could all be comprehensive results. However it does seem worthwhile to introduce students to the concept and have them do some simple examples so that those who proceed into mathematical careers (or careers that use mathematics) are more thorough and careful. The concept and ideas behind a Kahanian antiderivative also seem worthwhile, but we do not expect that to be as prevalent. 


\subsection{Adoption issues}

We are well aware that an abrupt transition to Kahanian parameterized antiderivatives is impossible. One of us implemented the non-piecewise generic portion of Kahan's example (2.4) in an early version of a computer algebra program. The complaints were immediate, numerous and strong. Even many mathematics teachers said that it was incorrect even though it differed only by a constant from the traditional antiderivative. Perhaps the space-saving omission of a generic integration constant $C$ from most integral tables led some mathematics teachers to forget what they taught, or perhaps they were simply concerned that it would alarm, and hence intimidate, many of their students. Another of us taught the concept to their beginning calculus students. They hated it.

\subsection{The pedagogical value of "lies to children"}

The Wikipedia entry on Lie-to-children quotes [11], "The pedagogical point is to avoid unnecessary burdens on the students first encounter with the concept." Asking students, on their first encounter with antiderivatives, to worry about the continuity of their answer, in addition to other worries, might seem unreasonable. Asking computer algebra systems to cater to the needs of first-year students as well as the needs of people who solve differential equations with parameters might also seem unreasonable, without some sort of switch to "expert mode", say. On the other hand, if only experts learn to be careful with parametric continuity, then both education and software have done a disservice to their audiences.

\subsection{Closing remarks}

In one sense this paper merely offers a minor technical correction to the current practice of computing indefinite integrals. However, the total impact of this minor correction is potentially large because the current practice is taught early at the university level and to very many students - most of whom do not go on to become mathematics majors. Moreover, computer algebra systems have become widespread, including good free ones, some of which are available for smartphones. Most current computer algebra systems apply current textbook rules and amplify the effects of fundamental "minor" errors such as the error in continuity that we address in this article. So in practice, the correction we present is important. 
In order to promote the ideas of comprehensive antiderivatives and Kahanian constants, we have developed a Mathematica program that computes comprehensive anti-derivatives and Kahanian forms. It can be used as an enhancement to Mathematica's own Integrate command. The program, as this paper, hopes to promote the analytical view common to several older calculus texts, such as those by Apostol [3] or Courant and John [8], over the more common current practice of making algebraic antidifferentiation the fundamental object of study.

The enhanced integration command is available on the website

https://www.uwo.ca/apmaths/faculty/jeffrey/research/integration/index.html

\section{References}

[1] Milton Abramowitz and Irene A. Stegun. Handbook of Mathematical Functions: with Formulas, Graphs, and Mathematical Tables. Dover, 1964.

[2] Robert A. Adams and Christopher Essex. Calculus, a complete course. Pearson, 8th edition, 2014.

[3] Tom M. Apostol. Mathematical Analysis: A Modern Approach to Advanced Calculus. Addison-Wesley, 1957.

[4] Carl M. Bender and Steven A. Orszag. Advanced Mathematical Methods for Scientists and Engineers. McGraw-Hill, New York, 1978.

[5] Manuel Bronstein. Symbolic Integration I. Transcendental Functions. Algorithms and Computation in Mathematics. Springer, 2nd edition, 2005.

[6] R. M. Corless and D. J. Jeffrey. Well... it isn't quite that simple. SIGSAM Bulletin, 26(3):2-6, 1992.

[7] Robert M. Corless, David J. Jeffrey, Stephen M. Watt, and James H. Davenport. "According to Abramowitz and Stegun" or arccoth needn't be uncouth. ACM SIGSAM Bulletin: Communications in Computer Algebra, 34(2):58-65, 2000.

[8] Richard Courant and Fritz John. Introduction to Calculus and Analysis I. Springer, 1999.

[9] Izrail Solomonovich Gradshteyn and Iosif Moiseevich Ryzhik. Table of Integrals, Series, and Products. Academic Press, 6th edition, 2007. 
[10] Thomas Hawkins. Weierstrass and the theory of matrices. Archive for History of Exact Sciences, 17(2):119-163, 1977.

[11] D. J. Jeffrey and Robert M. Corless. Teaching linear algebra with and to computers. In Wei-Chi Yang, editor, Proceedings of ATCM 2001, pages 120-129, 2001.

[12] D. J. Jeffrey and A.D. Rich. Reducing expression size using rulebased integration. In S. Autexier, editor, Intelligent Computer Mathematics, volume 6167 of LNAI, pages 234-246. Springer, 2010 .

[13] Harold Jeffreys and Bertha Swirles. Methods of Mathematical Physics. Cambridge University Press, 3rd edition, 1972.

[14] W. M. Kahan. Integral of $x^{n}$. Personal communication, circa 1976.

[15] H. L. Mencken. The divine afflatus. In Prejudices. Second Series, chapter IV, pages 155-171. Alfred A. Knopf, New York, 1920.

[16] Anatolii Platonovich Prudnikov, Yuri A. Brychkov, and Oleg Igorevich Marichev. Integrals and Series. CRC Press, 1992.

[17] Robert H. Risch. The solution of the problem of integration in finite terms. Bull. Amer. Math. Soc., 76(3):605-608, 1970.

[18] James Stewart. Calculus. Brooks Cole, 7th edition, 2012.

[19] Adrian Fedorovich Timofeev. Integrirovanie funktsii (Integration of Functions). Publishing House tehniko-teoreticheskoj literature, Leningrad, 1948.

[20] Daniel Zwillinger. Standard Mathematical Tables and Formulae. CRC Press, 32nd edition, 2011. 